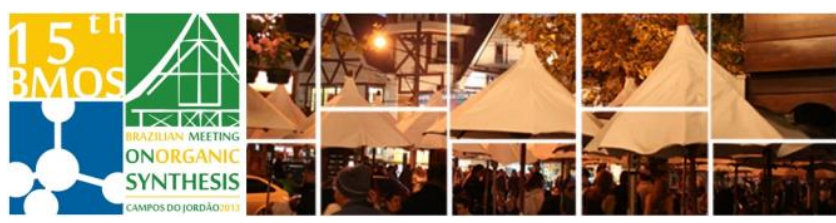

\title{
Organocatalysts: Design, Synthesis and Application in Stereoselective Domino-Michael/Henry Reactions.
}

\author{
Marcia Fulas, Jéssica de S. Figueiredo, Alessandro Rodrigues \\ ICAQF-UNIFESP, 09972-270, Diadema - SP, Brasil. \\ marcia.fulas@unifesp.br and alessandro.rodrigues@unifesp.br
}

Keywords: synthesis, catalysis, stereoselective.

\section{INTRODUCTION}

The stereoselective catalysis fits the principles of green chemistry, employing catalysts as selective as possible and replacing the usual stoichiometric reagents.

This work aims to design and develop a new organocatalysts class to apply in the synthesis of clausenamide stereoisomers.[1] Clausena lansium alkaloids isomers have been shown to be effective liver protectors, they are also traditionally employed in acute and chronic viral hepatitis, asthma, flu, gastrointestinal disorders and dermatological diseases treatment.[2]

\section{RESULTS AND DISCUSSION}

According to our retrosynthetic approach (Scheme 1) the synthesis of the clausenamide isomers could be expected from the key intermediate. We propose the domino nitro-Michael/Henry reaction for the synthesis of the key intermediate.

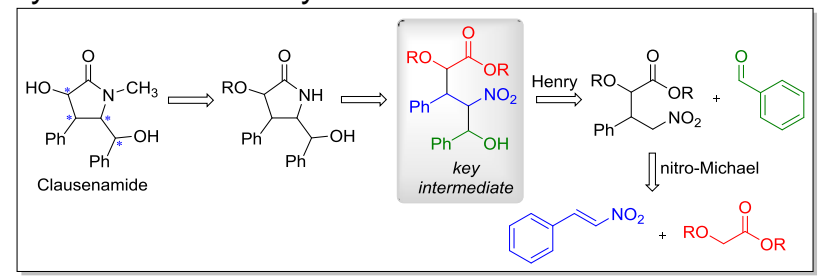

Scheme 1. Clausenamide retrosynthetic approach.

The first step implicated the design of the organocatalyst class. The optimum organocatalyst should be able to act enantioselectively in both nitroMichael and Henry reactions. Our organocatalysts involve the 1,8-diamino-anthracene as the scaffold.

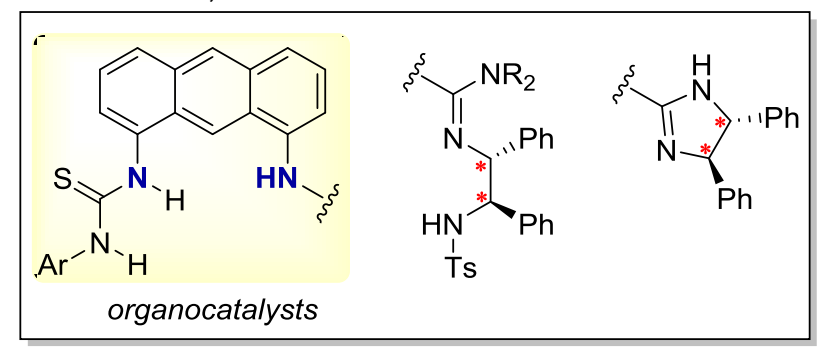

\section{Figure 1.}

The crucial aspect of this approach consists in the evaluation of the more stable conformations between catalyst and substrate, in order to verify if these combinations allow the reactants to assume a key geometry, favourable to the occurrence of the reaction. Catalysts candidates that do not meet these settings were discarded. A preliminary study indicated the thiourea and guanidine fragment groups as potential candidates (Figure 1). This stage consisted in the molecular modelling of bifunctional groups attached to the scaffold ideal for the dominonitro-Michael/Henry (Figure 2).

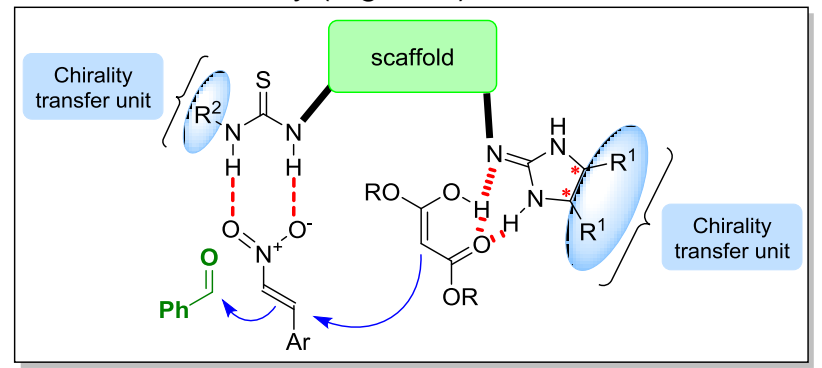

Figure 2.

The second step involves the experimental validation of the predicted catalysts. The 1,8diamino-anthracene scaffold was synthesized in two steps from 1,8-dinitroantraquinona in $83 \%$ yield. For the guanidine moiety, the isomers $R, R$ - and $S, S$ DPEN were obtained using standard procedures.[3] The next stage is to complete the organocatalysts synthesis and apply them for the stereoselective key intermediate synthesis.

\section{CONCLUSION}

The molecular modelling studies indicate that organocatalysts are candidates for the estereoselective domino nitro-Michael/Henry reaction. Studies are underway to confirm experimentally these predictions.

\section{ACKNOWLEDGEMENTS}

We thank the FAPESP for financial support. M.F thanks to CAPES for the scholarship. J.S.F. thanks to CNPq-PIBIC for the scholarship.

\section{REFERENCES}

${ }^{1}$ Hartwig, W.; Born, L. J. Org. Chem. 1987, 52, 4352-4358.

2 Yang, L.; Wang, D.-X.; Zheng, Q.-Y.; Pan, J.; Huang, Z.-T.; Wang, M.-X. Org. Biomol. Chem. 2009, 7, 2628-2634.

${ }_{3}^{3}$ Pikul, S.; Corey, E. J. Org. Synth. 1993, 71, $22-29$.

\footnotetext{
$15^{\text {th }}$ Brazilian Meeting on Organic Synthesis - 15 $5^{\text {th }}$ BMOS - November 10-13, 2013 - Campos do Jordão, Brazil
} 